\title{
Quadro clínico de mulheres acometidas por acidente vascular cerebral em uso de
}

\section{anticoncepcionais hormonais}

\author{
The clinical condition of women affected by stroke using hormonal contraceptives \\ Cuadro clínico de las mujeres afectadas por un accidente cerebrovascular que usan anticonceptivos
}

hormonales

Recebido: 17/06/2021 | Revisado: 25/06/2021 | Aceito: 02/07/2021 | Publicado: 14/07/2021

\author{
Larissa Mariana Oliveira Santos Batista \\ ORCID: https://orcid.org/0000-0002-7972-4444 \\ Universidade Estadual de Campinas, Brasil \\ E-mail: larimariana.santos@gmail.com \\ Maria Helena de Melo Lima \\ ORCID: https://orcid.org/0000-0001-6521-8324 \\ Universidade Estadual de Campinas, Brasil \\ E-mail: melolima@unicamp.br \\ Ana Railka de Souza Oliveira-Kumakura \\ ORCID: https://orcid.org/0000-0002-7075-7987 \\ Universidade Estadual de Campinas, Brasil \\ E-mail: arailka@unicamp.br
}

\begin{abstract}
Resumo
Objetivo: investigar o quadro clínico de mulheres em uso de anticoncepcionais hormonais e que foram acometidas por acidente vascular cerebral (AVC). Método: revisão de escopo mediante estratégia de busca em quatro bases de dados (Pubmed, CINAHL, Scopus e Web of Science). Critérios de inclusão: estudos quantitativos ou qualitativos, estudos de revisão e literatura cinzenta, nos idiomas português, inglês ou espanhol nos últimos 10 anos. Excluíram-se editoriais e artigos que não respondiam à questão norteadora. Os dados foram apresentados na forma de tabelas $\mathrm{e}$ categorias de análise que permitiram sintetizar de forma narrativa os achados. Resultados: Identificados cinco estudos. Anticoncepcionais oral combinado, injetável combinado e de emergência foram os mais utilizados, mas nem sempre os artigos destacam a posologia, via de utilização e tempo de uso prévio. As pacientes foram avaliadas com uso de escalas e exame físico, da admissão até a reabilitação. A sobrevida das pacientes foi alta, mas a taxa de mortalidade e risco de recorrência eram elevados. Conclusão: o AVC em mulheres usuárias de anticoncepcionais foi tão grave quanto nas não usuárias, e aquelas que usaram o anticoncepcional por menor tempo apresentaram menor gravidade do AVC.
\end{abstract}

Palavras-chave: Acidente Vascular Cerebral; Anticoncepcionais; Sinais e sintomas; Evolução clínica; Enfermagem.

\begin{abstract}
Aim: To investigate the clinical condition of women using hormonal contraceptives and who were affected by stroke. Method: Scope review using a search strategy in four databases (Pubmed, CINAHL, Scopus, and Web of Science). The inclusion criteria were: quantitative or qualitative studies, review studies, and gray literature in Portuguese, English, or Spanish in the last 10 years. Editorials and articles that do not answer the guiding question were excluded. The data were shown in the form of tables and categories of analysis that allowed to synthesize the findings narratively. Results: The samples included five articles. Combined oral, combined injectable and emergency contraceptives were the most used, but not always highlighted the dosage, route of use, and time of the previous usage. To evaluation of the woman used scales and physical examination, from admission to rehabilitation. The patients' survival was high. Conclusion: The stroke in women using contraceptives was as severe as in non-users, and those who used the contraceptive for a shorter time had a less severe stroke.
\end{abstract}

Keywords: Stroke; Contraceptives agents; Signs and symptoms; Clinical evolution; Nursing.

\section{Resumen}

Objetivo: investigar el cuadro clínico de las mujeres que usan anticonceptivos hormonales y que fueron afectadas por un accidente cerebrovascular. Método: revisión del alcance utilizando una estrategia de búsqueda en cuatro bases de datos (Pubmed, CINAHL, Scopus y Web of Science). Se han incluido estudios cuantitativos o cualitativos, estudios de revisión y literatura gris en portugués, inglés o español en los últimos 10 años. Se excluyeron editoriales y artículos que no responden a la pregunta guía. Los datos se presentaron en forma de tablas y descripciones, y se construyeron categorías de análisis que permitieron sintetizar los hallazgos de forma narrativa. Resultados: Se identificaron cinco estudios. Referente a los métodos anticonceptivos orales combinados, los inyectables combinados y la anticoncepción 
de emergencia oral fueron los más utilizados, pero no siempre se reportaron la dosis, la ruta de uso y el tiempo de uso anterior. Los pacientes fueron evaluados mediante escalas y examen físico, desde el ingreso hasta la rehabilitación. La supervivencia de los pacientes fue alta. Conclusión: el derrame cerebral en las mujeres que usaron anticonceptivos fue tan severo como en las no usuarias, y aquellas que usaron el anticonceptivo por un tiempo más corto tuvieron un derrame cerebral menos severo.

Palabras clave: Accidente Cerebral Vascular; Anticonceptivos; Signos y síntomas; Evolución clínica; Enfermería.

\section{Introdução}

O acidente vascular cerebral (AVC) é uma das principais causas de morte por doenças não transmissíveis e, portanto, pesquisas têm sido realizadas para investigar as características específicas de cada sexo e a ocorrência deste fenômeno (Madsen et al., 2019; Ministério da Saúde, 2018; Pang, Fu, Cao, Hao, \& Zong, 2019; Poorthuis, Algra , Algra, Kappelle, \& Klijn, 2017). De acordo com informações divulgadas pelo Ministério da Saúde do Brasil, a taxa de óbito por AVC em brasileiras entre 30 e 69 anos, no período de 2010 a 2016, caiu 11\%, aumentando, portanto, a população que possivelmente frequentará ambulatórios e clínicas de reabilitação no país, e que, portanto, demandarão cuidados de uma equipe de enfermagem treinada (Ministério da Saúde, 2018).

Para as mulheres em idade fértil, o uso de anticoncepcionais hormonais combinados contendo estrogênio e progesterona é um dos fatores de risco bem estabelecidos para a ocorrência de AVC (Champaloux et al., 2017; Poorthuis, Algra, Algra, Kappelle, \& Klijn, 2017; Xu, Li, Tang, Huang, \& Chen, 2015). Além do evento cerebrovascular, o uso deste medicamento pode aumentar o risco para outros eventos tromboembólicos, como, trombose venosa profunda, tromboembolismo pulmonar e infarto do miocárdio (Chang et al., 2017; Hugon-Rodin, Gompel, \& Plu-Bureau, 2017; Xu et al., 2018).

Uma revisão sistemática revelou que há risco aumentado de 1,6 para o AVC isquêmico ou infarto do miocárdio entre mulheres que utilizam anticoncepcionais orais combinados (AOC), com o maior risco no uso de pílulas com > 50 $\mu \mathrm{g}$ de estrogênio (Roach et al., 2015). O AVC, quando não relacionado ao método anticoncepcional, não é comum em mulheres antes da menopausa, havendo um aumento da incidência com o avanço da idade, sobretudo após os 75 anos (Barker-Collo et al., 2015; Hugon-Rodin, Gompel, \& Plu-Bureau, 2017).

O uso de anticoncepcionais hormonais é um dos métodos mais utilizados para o controle de natalidade em todo o mundo. Estima-se que em vários países mais de $80 \%$ das mulheres utilizaram este método em algum momento de sua vida reprodutiva (Hugon-Rodin, Gompel, \& Plu-Bureau, 2017). O estrogênio, além de causar disfunções endoteliais, altera significativamente o sistema de coagulação, aumentando a trombina e os fatores de coagulação, favorecendo o desenvolvimento de eventos tromboembólicos, sobretudo em mulheres que já possuem outras pré-disposições clínicas como, hipertensão, tabagismo, migrânea e dislipidemia (Lima et al., 2017a). Mesmo em baixa dosagem, diferentes tipos de associação com progestágenos e distintos tempos de uso, as mulheres que fazem uso de AH apresentam maior risco para o AVC, porém isso pode aumentar ou diminuir com a variação desses aspectos, sendo o risco sempre maior do que em mulheres que não fazem o uso do medicamento (Lima et al., 2017a; Peters et al., 2015).

Apesar da clareza na literatura acerca da relação entre o uso de anticoncepcionais hormonais combinado e a ocorrência do AVC, pouco se sabe sobre a evolução clínica dessas mulheres após o diagnóstico da doença. Um estudo de revisão integrativa que pesquisou a influência do uso desses medicamentos na ocorrência do AVC encontrou apenas uma publicação que demonstrou o aumento na taxa de morbimortalidade e incapacidades nas usuárias de AOC de segunda geração, mas nela não havia comparação entre usuárias e não-usuárias do medicamento (Lima et al., 2017a).

Portanto, considerando o crescente uso dos anticoncepcionais em todo o mundo, o aumento significativo da morbidade após o AVC e a ocorrência deste evento em mulheres mais jovens, é necessário que os profissionais de saúde compreendam qual a relação dos fatores de risco com o prognóstico das pacientes, visto que, somente conhecendo esta relação 
será possível direcionar as medidas de prevenção aos fatores de risco modificáveis, sobretudo na atenção básica em que a atuação do enfermeiro em práticas de educação em saúde é tão presente.

Com isso, o objetivo dessa revisão de escopo foi investigar o quadro clínico de mulheres em uso de anticoncepcionais hormonais após o episódio de AVC. Dessa forma buscou-se sobre os sinais e sintomas de admissão e alta, avaliação neurológica, taxa de mortalidade e morbidade. A hipótese deste estudo é que apesar da alta prevalência para o desenvolvimento de $\mathrm{AVC}$, o prognóstico da doença não é diferente entre as usuárias ou não deste tipo de medicação.

\section{Metodologia}

Estudo de revisão de escopo realizado para fornecer uma visão geral da base das evidências existentes, independentemente da qualidade metodológica dos estudos incluídos. Dessa forma, permite uma revisão exaustiva de literatura complexa e heterogênea, em que não é possível a realização de uma revisão sistemática mais precisa (Peters et al., 2015).

Utilizando a estratégia de População, Conceito e Contexto para a formulação da questão de pesquisa do estudo: a) como população: mulheres em uso de contraceptivos hormonais após o diagnóstico de AVC; b) como conceito: estudos com foco no quadro clínico das mulheres em relação à mortalidade, morbidade e evolução neurológica; c) como contexto: todas as configurações clínicas (internação e reabilitação) e geográficas (Peters et al., 2015). O objetivo foi responder a seguinte questão: como é o quadro clínico de mulheres em uso de anticoncepcionais hormonais após o episódio de AVC?

Para a busca na literatura, foram acessadas num único dia, no mês de setembro de 2019, as bases de dados US National Library of Medicine (PUBMED), Cumulative Index to Nursing \& Allied Health Literature (CINAHL), Scopus Info Site (SCOPUS) e Web of Science. Utilizou-se terminologia preconizada para cada base: PUBMED/vocabulário Medical Subject Headings (MeSH); CINAHL/TÍTULO. Como as bases SCOPUS e Web of Science não possuem vocabulário próprio, foi utilizada a terminologia MeSH. A busca em cada base foi realizada por duas pesquisadoras e teve suporte de uma bibliotecária. Além disso, procedeu-se a leitura das referências dos artigos incluídos para incluir o maior número de fontes que respondessem à questão norteadora. O Quadro $1 \mathrm{em}$ anexo apresenta as estratégias de busca adotadas.

Critérios de inclusão: estudos quantitativos ou qualitativos, estudos de revisão, bem como material não publicado (literatura cinzenta), nos idiomas português, inglês ou espanhol nos últimos 10 anos. Adotou-se esse recorte temporal dada a publicação de uma revisão integrativa da literatura, apresentada anteriormente, que também buscou compreender essa relação, mas que encontrou apenas uma publicação que respondesse sobre a evolução clínica das mulheres (Lima et al., 2017a). Excluídas as produções que não responderam à questão norteadora e os editoriais.

As buscas foram exportadas para o software on-line EndNote ${ }^{\mathrm{TM}}$ software (Clarivate Analytics) que permitiu o gerenciamento das referências. Os títulos e resumos foram lidos e analisados por duas revisoras independentes. Em caso de dúvidas, foi realizada a leitura completa do material para verificar a pertinência do texto à revisão. Duas revisoras realizaram a extração dos dados das referências incluídas, que contemplou: título, ano de publicação, autores, revista de publicação, país do estudo, objetivos do estudo, desenho metodológico, tamanho da amostra, período do estudo, forma de acompanhamento das pacientes, utilização de escalas, apresentação de exames, registro dos sinais e sintomas, o que foi avaliado (sinais e sintomas, severidade, morbidade e mortalidade), características do anticoncepcional, resultados, limitações apresentadas pelo estudo, considerações finais e nível de evidência. Os desacordos foram resolvidos por discussões durante cada etapa.

Os estudos receberam um código para facilitar a identificação e apresentação dos resultados (E1, E2, E3...). Para a classificação do nível de evidência, foram adotadas as seguintes categorias: nível 1 - evidências de revisão sistemática ou metanálise de ensaios clínicos randomizados controlados ou diretrizes clínicas baseadas em revisões sistemáticas de ensaios clínicos randomizados controlados; nível 2 - evidências de ensaio clínico randomizado controlado; nível 3 - evidências de ensaios clínicos sem randomização; nível 4 - evidências de estudos de coorte e de caso-controle; nível 5 - evidências de revisão 
sistemática de estudos descritivos e qualitativos; nível 6 - evidências de único estudo descritivo ou qualitativo; nível 7 evidências de opinião de autoridades e/ou relatório de comitês de especialistas (Melnyk \& Fineout-Overholt, 2005). Salienta-se que não foram utilizados estudos para fazer a análise da qualidade metodológica dos artigos incluídos nesta revisão.

Os resultados dos estudos foram apresentados na forma de quadro e analisados de forma narrativa. Para sintetizar os achados acerca do quadro clínico das mulheres acometidas por AVC e que estavam em uso de ACO, foram construídas três categorias de análise, que emergiram do conteúdo analisado. Como salientado por estudo prévio, para a construção dessas categorias utilizou-se a frequência de aparecimento do tema e a relevância implícita (Campos, 2004).

\section{Resultados}

Após a leitura dos títulos e resumos dos artigos selecionados, e posteriormente dos artigos completos, a amostra final foi composta por cinco estudos que foram selecionados por preencherem os critérios de inclusão. O fluxograma do processo de busca e seleção dos estudos dessa revisão, segundo checklist adaptado do Preferred Reporting Items for Systematic Reviews and Meta-Analyses (PRISMA) (Lillemoen \& Pedersen, 2013), está apresentado na Figura 1.

Figura 1 - Fluxograma do processo de identificação, seleção, elegibilidade e inclusão dos estudos baseado nas recomendações do PRISMA.

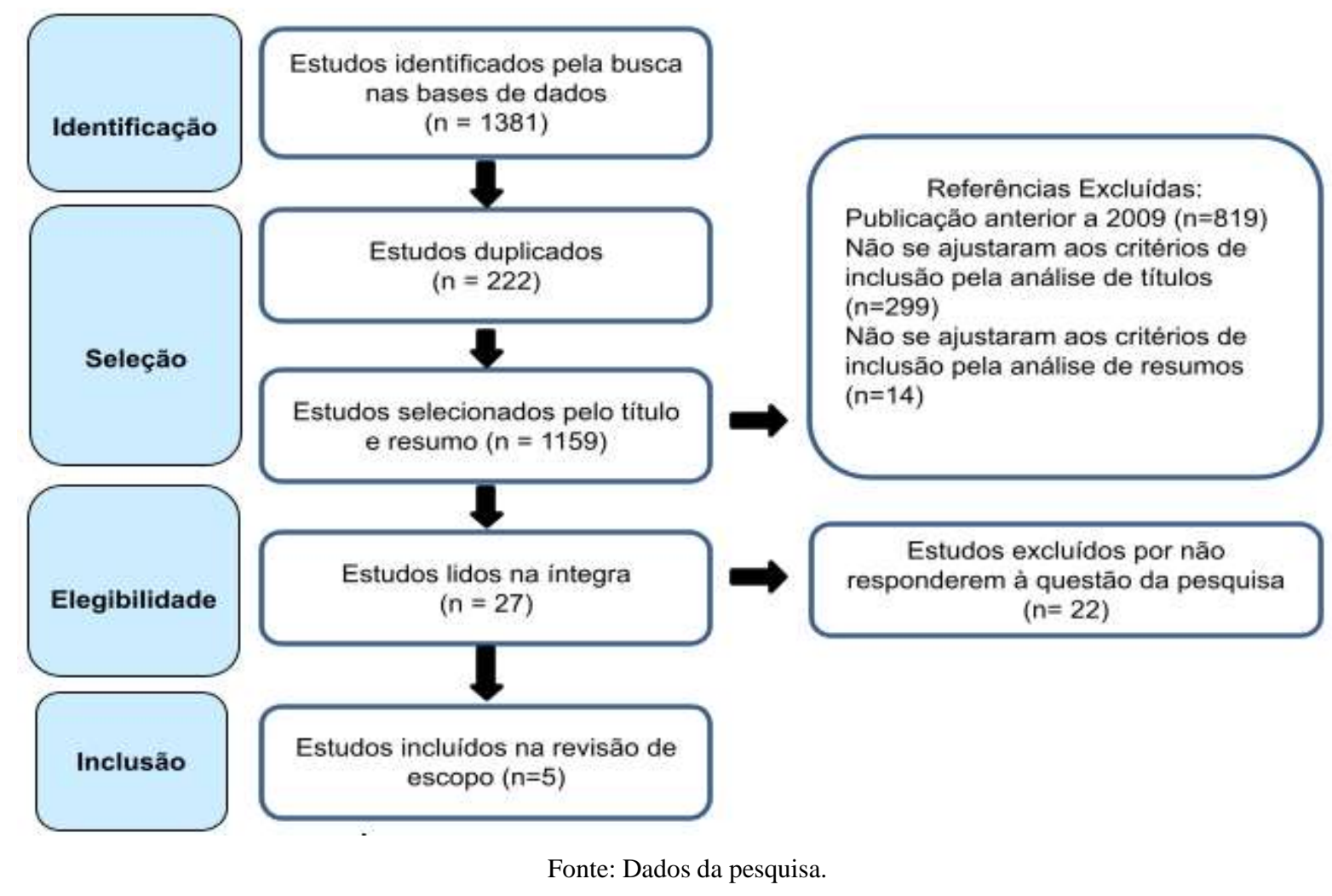

Os artigos incluídos na revisão foram publicados entre os anos de 2011 (Antonio, Fuentes, Aguilar-Amat, \& DíezTejedor, 2011) e 2017 (Lima et al., 2017b), em países europeus (Andersen \& Olsen, 2014; Antonio, Fuentes, Aguilar-Amat, \& Díez-Tejedor, 2011; Maino, Siegerink, Algra, Peyvandi \& Rosendaal, 2016), nos Estados Unidos (Selvan et al., 2017) e no Brasil (Lima et al., 2017b). Duas das publicações são estudos do tipo caso controle (Andersen \& Olsen, 2014; Maino, Siegerink, Algra, Peyvandi \& Rosendaal, 2016), as outras três se diferenciam em relato de caso (Antonio, Fuentes, AguilarAmat, \& Díez-Tejedor, 2011), revisão sistemática (Selvan et al., 2017) e estudo de coorte (Lima et al., 2017b). A amostra incluída nos estudos variou de 1 a 618, totalizando 903 mulheres em uso de AOC que foram acompanhadas. 
Predominantemente os estudos foram publicados em revistas médicas, porém um estudo publicado em uma revista direcionada à farmacoepidemiologia e outro estudo na área de enfermagem. Apenas um estudo possui nível de evidência 1 (Selvan et al., 2017), três se enquadraram no nível 4 (Andersen \& Olsen, 2014; Lima et al., 2017b; Maino, Siegerink, Algra, Peyvandi \& Rosendaal, 2016) e um no nível de evidência 6 (Antonio, Fuentes, Aguilar-Amat, \& Díez-Tejedor, 2011).

Quadro 2 - Características dos estudos selecionados. Campinas, 2019.

\begin{tabular}{|c|c|c|c|c|}
\hline Título e Autores & $\begin{array}{l}\text { Revista, ano e país de } \\
\text { publicação }\end{array}$ & $\begin{array}{c}\text { Tipo de estudo } \\
\text { e tamanho } \\
\text { amostral }\end{array}$ & $\begin{array}{c}\text { Anticoncepcional } \\
\text { utilizado }\end{array}$ & $\begin{array}{l}\text { Critérios de } \\
\text { avaliação das } \\
\text { usuárias }\end{array}$ \\
\hline $\begin{array}{l}\text { E1. "Píldora del día después" e ictus: un nuevo } \\
\text { caso Emergency contraception and stroke. A } \\
\text { new case report. }\end{array}$ & $\begin{array}{l}\text { Medicina Clínica } \\
2011 \\
\text { Espanha }\end{array}$ & $\begin{array}{l}\text { Relato de caso } \\
\qquad \mathrm{N}=1\end{array}$ & $\begin{array}{l}\text { Emergência } \\
\text { (combinado) }\end{array}$ & $\begin{array}{l}\text { Déficit neurológico } \\
\text { pelos indicadores da } \\
\text { National Institute of } \\
\text { Health Stroke Scale e } \\
\text { da Escala de Rankin } \\
\text { Modificada }\end{array}$ \\
\hline $\begin{array}{l}\text { E2. Severity of Stroke in Women Using Oral } \\
\text { Contraceptives. }\end{array}$ & $\begin{array}{c}\text { Pharmacoepidemiology and } \\
\text { Drug Safety } \\
2014 \\
\text { Dinamarca }\end{array}$ & $\begin{array}{c}\text { Caso Controle } \\
\text { Caso }=618 \\
\text { Controle }=1746\end{array}$ & $\begin{array}{c}\text { Informado apenas } \\
\text { que foi } \\
\text { anticoncepcional } \\
\text { hormonal }\end{array}$ & $\begin{array}{l}\text { Déficit neurológico } \\
\text { pelos critérios da } \\
\text { Scandinavian } \\
\text { Stroke Scale }\end{array}$ \\
\hline $\begin{array}{c}\text { E3. Recurrence and mortality in young women } \\
\text { with myocardial infarction or ischemic stroke: } \\
19 \text {-year follow-up of the risk of arterial } \\
\text { thrombosis in relation to oral contraceptives } \\
\text { (RATIO) study. }\end{array}$ & $\begin{array}{l}\text { JAMA Internal Medicine } \\
2015 \\
\text { Holanda }\end{array}$ & $\begin{array}{c}\text { Caso Controle } \\
\text { Caso }=160 \\
\text { Controle }=782\end{array}$ & $\begin{array}{c}\text { Informado apenas } \\
\text { que foi } \\
\text { anticoncepcional } \\
\text { hormonal }\end{array}$ & $\begin{array}{l}\text { Morbidade e } \\
\text { mortalidade }\end{array}$ \\
\hline $\begin{array}{l}\text { E4. Stroke and Etonogestrel/Ethinyl Estradiol } \\
\text { Ring (NuvaRing): Clinical, Radiological, and } \\
\text { Prognostic Features. }\end{array}$ & $\begin{array}{c}\text { Journal of Stroke and } \\
\text { Cerebrovascular Diseases } \\
2016 \\
\text { Estados Unidos }\end{array}$ & $\begin{array}{l}\text { Revisão } \\
\text { Sistemática } \\
\text { N=19 }\end{array}$ & $\begin{array}{l}\text { Anel vaginal com } \\
\text { hormônios } \\
\text { combinados }\end{array}$ & $\begin{array}{l}\text { Sinais e sintomas, } \\
\text { achados radiológicos, } \\
\text { mortalidade e } \\
\text { morbidade }\end{array}$ \\
\hline $\begin{array}{l}\text { E5. Efeitos do uso de anticoncepcionais } \\
\text { hormonais combinados sobre o acidente } \\
\text { vascular cerebral. }\end{array}$ & $\begin{array}{c}\text { Tese (doutorado) } \\
2017 \\
\text { Brasil }\end{array}$ & \begin{tabular}{|c|} 
Estudo de \\
coorte \\
$\mathrm{N}=105(37$ \\
usuárias, 68 não \\
usuárias)
\end{tabular} & $\begin{array}{c}\text { Combinado oral e } \\
\text { injetável }\end{array}$ & $\begin{array}{c}\text { Déficit neurológico } \\
\text { pelos critérios da } \\
\text { National Institute of } \\
\text { Health Stroke Scale, } \\
\text { Escala de Rankin } \\
\text { Modificada e Índice } \\
\text { de Barthel }\end{array}$ \\
\hline
\end{tabular}

Fonte: dados da pesquisa.

\section{Categoria 1 - Tipos de anticoncepcionais hormonais e o acidente vascular cerebral}

Apesar do uso de anticoncepcionais hormonais combinado, nem todos os estudos apresentaram a concentração do princípio ativo ou a via de administração do medicamento utilizado (Andersen \& Olsen, 2014; Maino, Siegerink, Algra, Peyvandi \& Rosendaal, 2016). Em E1 apresenta-se o caso de uma paciente que usou um anticoncepcional de emergência e, 
mesmo sem outros fatores de risco evidentes, apresentou o quadro de AVC (Antonio, Fuentes, Aguilar-Amat, \& Díez-Tejedor, 2011).

O E4 (Maino, Siegerink, Algra, Peyvandi \& Rosendaal, 2016) trata-se de uma revisão sistemática que abordou o uso do anel vaginal por 19 mulheres, com dosagem de $120 \mu \mathrm{g}$ de etonogestrel (progestina esteróide de terceira geração) e $15 \mu \mathrm{g}$ de estradiol (estrogênio esteróide), considerado um anticoncepcional hormonal combinado de baixa dose, com menos

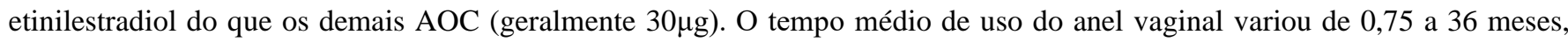
com média de 11,2 (DP = 11,1) sendo que cinco dos casos $(26,3 \%)$ usavam simultaneamente outra forma de anticoncepcional hormonal.

O método anticoncepcional predominante no E5 (Lima et al., 2017b) foi o AOC. Porém, uma paciente relatou o uso do anticoncepcional hormonal injetável combinado. Verificou-se que o etinilestradiol foi o único estrógeno, e os progestágenos variaram, sendo o levonorgestrel o mais prevalente, (73,7\%). Em relação à dosagem hormonal, 83,8\% das usuárias utilizavam

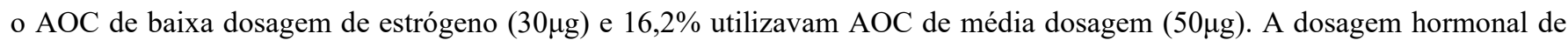
progestágeno variou de $75 \mu \mathrm{g}$ a $3000 \mu \mathrm{g}{ }^{19}$.

Além disso, no estudo E5 (Lima et al., 2017b) foi realizada a comparação entre a maior e menor dosagem hormonal com o perfil clínico das usuárias. Mulheres com alteração do nível de consciência e desequilíbrio faziam o uso de AOC com maior dosagem de estrógeno $(0,05 \mathrm{mg})$. A dosagem de progestágeno foi mais elevada $(3 \mathrm{mg})$ entre as mulheres que tinham déficit motor, alteração do nível de consciência, de visão, desequilíbrio e maior gravidade, conforme resultado na National Institute of Health Stroke Scale na avaliação inicial. Menor tempo de uso do anticoncepcional foi verificado entre as mulheres com menor gravidade do AVC, porém pelo desenho deste estudo não é possível estabelecer se as alterações ateroscleróticas e as mudanças na função e estrutura endotelial, causadas pelo uso prolongado do AOC, contribuíram para maior comprometimento neurológico.

\section{Categoria 2 - Quadro clínico na admissão e forma de acompanhamento}

No relato de caso E1 (Antonio, Fuentes, Aguilar-Amat, \& Díez-Tejedor, 2011), os autores registraram os seguintes sinais e sintomas na admissão da paciente: migrânea sem aura, hemiparesia à direita, afasia, diplopia e hipoestesia hemifacial direita. Contudo, não houve aplicação de escalas neurológicas na admissão da paciente.

Nos estudos de caso controle (Andersen \& Olsen, 2014; Maino, Siegerink, Algra, Peyvandi \& Rosendaal, 2016), os autores realizaram o acompanhamento das pacientes a partir dos registros nacionais dos casos de AVC (E2) (Andersen \& Olsen, 2014) e registros nacionais de atestados de óbito e registros de dados hospitalares (E3) (Maino, Siegerink, Algra, Peyvandi \& Rosendaal, 2016) respectivamente. Não há registro de aplicação de escalas de avaliação neurológica nas 160 pacientes de E3, porém no E2 os autores analisaram a pontuação das 2.364 pacientes na Scandinavian Stroke Scale, não especificando o momento de sua aplicação. As pacientes em uso de anticoncepcional hormonal tiveram uma pontuação mediana de 48,0 na Scandinavian Stroke Scale, não diferindo estatisticamente das pacientes com AVC e sem o uso do medicamento, que pontuaram 48,3, o que representa baixo risco de comprometimento neurológico. Após análise da mediana da pontuação da escala e realização de ajuste na idade, tipo de AVC, posição socioeconômica e tabagismo atual, verificou-se que o AVC em mulheres usuárias de anticoncepcional foi tão grave quanto o AVC em mulheres que não são usuárias da medicação.

A avaliação das 19 pacientes de E4 (Selvan et al., 2017) ocorreu pelo acompanhamento dos sinais e sintomas apresentados na admissão das pacientes, não havendo aplicação de escalas neurológicas. Neste estudo a idade das mulheres variou de 14 a 48 anos, com uma média de 31,7 anos (DP =9,8). Em relação às manifestações clínicas no momento da admissão, 36,8\% das usuárias queixaram-se de dor de cabeça, 36,8\% fraqueza motora e 21,0\% apresentaram convulsões. 
No estudo E5 (Lima et al., 2017b) realizou-se o seguimento de 105 pacientes, com uma avaliação inicial na internação e acompanhamento após três meses do AVC, por meio de ligação telefônica. A verificação do comprometimento neurológico, da gravidade do AVC, da funcionalidade e do grau de dependência foi realizada a partir da identificação dos déficits apresentados (motor, sensibilidade, nível de consciência, fala, visual e desequilíbrio) e da aplicação das seguintes escalas de avaliação neurológica: National Institute of Health Stroke Scale, Escala Rankin Modificada e Índice de Barthel Modificado, com repetição das duas últimas no contato telefônico após três meses. Quanto à identificação dos déficits na avaliação inicial, 68.6\% das mulheres em uso de anticoncepcional apresentaram déficit motor, 51,4\% déficit de fala, 31,4\% déficit de sensibilidade, 31,4\% déficit visual, $20 \%$ desequilíbrio e 17,1\% alteração no nível de consciência. A média das escalas na avaliação inicial foi de 5,3 (DP=6,1) na National Institute of Health Stroke Scale (déficit moderado), 2,9 (DP=1,7) na Escala Rankin Modificada (incapacidade moderada) e 34,3 (DP=13,6) no Índice de Barthel Modificado (moderada dependência).

\section{Categoria 3 - Quadro clínico na alta e desfechos}

No relato de caso (E1) (Antonio, Fuentes, Aguilar-Amat, \& Díez-Tejedor, 2011), os autores indicaram que duas escalas foram utilizadas para avaliação da paciente no momento da alta: National Institute of Health Stroke Scale e Escala Rankin Modificada. Os autores descrevem que a evolução neurológica da paciente foi favorável, visto que no momento da alta apresentava apenas um déficit sensitivo, apresentando a nota 1 nas duas escalas, indicativo de baixa severidade do AVC e baixo comprometimento funcional.

Em E2 (Andersen \& Olsen, 2014) não há apresentação de dados referentes à alta das pacientes ou taxas de mortalidade.

O estudo holandês (E3) (Maino, Siegerink, Algra, Peyvandi \& Rosendaal, 2016) fez o acompanhamento de pacientes usuárias de anticoncepcional com o objetivo de verificar o risco para ocorrência de novos acidentes vasculares cerebrais, eventos cardiovasculares e avaliou as taxas de mortalidade. A mortalidade elevada persistiu ao longo do tempo, em pacientes que tiveram o AVC em uso do anticoncepcional, comparando com as que não tiveram o evento, e foi principalmente apoiada por uma elevada taxa de mortes por eventos vasculares agudos. Eventos cardiovasculares fatais e não fatais foram mais frequentes nas pacientes que tiveram AVC do tipo isquêmico. Nas pacientes com AVC, a taxa de novos eventos cerebrais foi de 11,1 por 1.000 pessoas/ano (IC 95\%, 7,5-15,9), enquanto o risco de eventos cardíacos foi de 2,7 por 1.000 pessoa/ano (IC $95 \%, 1,2-5,4)$.

Por outro lado, a revisão sistemática (E4) (Selvan et al., 2017) identificou que os prognósticos das 19 pacientes avaliadas eram bons, com sobrevida de $95 \%$, sendo que cinco foram para reabilitação, quatro para clínicas de atendimento de enfermagem especializada, nove para casa e uma faleceu. A paciente que faleceu era a única que tinha em seu histórico pessoal a paralisia cerebral e foi a única que apresentou, na admissão, alteração do estado mental. Entre as pacientes que tiveram alta, as manifestações clínicas na admissão variaram entre migrânea, cefaleia, convulsão, vertigem e hemiplegia à direita. Entre as mulheres encaminhadas para reabilitação ou clínicas de atendimento de enfermagem especializada, além dos sinais e sintomas já citados, registou-se também na admissão tontura e afasia. Não houve solicitação de exame neurológico nas altas, sendo esta uma limitação apresentada pelo estudo.

No estudo E5 (Lima et al., 2017b) foi possível notar que após 3 meses do AVC, muitas mulheres apresentaram remissão total dos déficits neurológicos, porém houve persistência do déficit de sensibilidade em 8,6\%, do déficit de fala em 11,4\%, déficit visual em 14,3\% e desequilíbrio em 5,7\%. O déficit motor foi o que continuou presente na maior parcela das mulheres (40\%). Nesta segunda avaliação também se realizou a aplicação primeiro da Escala de Rankin Modificada, com pontuação de 1,5 ( $\mathrm{DP}=1,5)$, o que representou de independência a incapacidade moderada. Em segundo momento avaliou-se 
o resultado fornecido pelo e Índice de Barthel Modificado que foi 46,5 (DP = 8,9), ou seja, variou de moderada dependência a independência.

\section{Discussão}

Esta revisão apresenta uma perspectiva global e recente sobre estudos que analisaram por meio de escalas e/ou exame físico a evolução do quadro clínico de mulheres que usavam anticoncepcional e desenvolveram um AVC.

Notou-se lacuna de estudos dedicados à avaliação da relação entre o uso dos anticoncepcionais e a gravidade do AVC. A maioria das publicações estuda os diferentes tipos de medicamento e o risco do seu uso para o desencadeamento do AVC. $\mathrm{Na}$ literatura atual há muitos artigos que abordam o anticoncepcional como um fator de risco, mas não há evidências fortes de que seu uso prévio modificaria o prognóstico clínico de suas usuárias (Chang et al., 2017; Salisbury, Pfeffer, \& Yip, 2011; Xu et al., 2018; Xu, Li, Tang, Huang, \& Chen, 2015).

Apesar de pesquisas apontarem o declínio das taxas de mortalidade por AVC no Brasil e no mundo durante as últimas décadas, elas também indicam o aumento da incidência do evento e destacam características específicas de cada sexo que aumentam o risco de AVC, sendo o uso do anticoncepcional uma delas (Lotufo et al., 2015; Ministério da Saúde, 2018; Vangen-Lønne et al., 2017)

Dois estudos incluídos na presente revisão de escopo mostraram que nas mulheres usuárias de ACO as taxas mortalidade eram elevadas, bem como a chance para desenvolver novos eventos cerebrais ou cardíacos (Maino, Siegerink, Algra, Peyvandi \& Rosendaal, 2016) e que mesmo apesar disso, a taxa de sobrevida era alta e o prognóstico era bom (Selvan et al., 2017).

Nesse sentido, é importante que o enfermeiro faça uma avaliação minuciosa das mulheres durante as consultas de planejamento familiar para identificar os antecedentes pessoais e familiares e, a partir disso, determinar os riscos e benefícios que o método apresenta para a mulher e diminuir a chance de um possível AVC ou outros eventos tromboembólicos (Couto et al., 2020).

Apesar da evolução de quem usou ou não anticoncepcionais hormonais sejam parecidas, devemos trabalhar para prevenir a ocorrência do AVC como apresentado em estudo de revisão sistemática (Appelros, Stegmayr, \& Terent, 2009) que investigou as diferenças sexuais relacionadas ao AVC. Nele identificou-se que os episódios isquêmicos são mais graves em mulheres, bem como a letalidade em um mês, sendo essa uma das justificativas para se aprofundar nos estudos acerca da evolução neurológica.

O primeiro ponto é que nem todos os estudos detalharam o anticoncepcional utilizado (Andersen \& Olsen, 2014; Maino, Siegerink, Algra, Peyvandi \& Rosendaal, 2016). No Brasil, o acesso aos anticoncepcionais pode ocorrer por dois caminhos: dispensação gratuita nas unidades básicas de saúde ou compra direta nas drogarias, onde não há controle para sua venda. Artigo publicado em 2017 demonstrou o aumento no faturamento dos laboratórios farmacêuticos com as vendas desses medicamentos nos últimos anos, o que ressalta a importância do aprofundamento das pesquisas sobre o uso de forma segura (Brandão \& Cabral, 2017).

Tal identificação possibilita a compreensão do cenário em que as medidas de educação em saúde poderão ser implantadas pela enfermagem, sejam para conscientização sobre o uso seguro dos fármacos, como por exemplo em palestras de educação sexual, ou, na educação individual durante as consultas de enfermagem para planejamento familiar (Couto et al., 2020). Além disso, convém destacar a importância do registro das informações durante a etapa de coleta de dados, pois o conhecimento de dados da anticoncepção servirá para melhor compreensão dos seus efeitos a curto e longo prazo por toda a equipe de saúde. 
Revisão sistemática evidenciou risco aumentado de AVC isquêmico ou infarto agudo do miocárdio em mulheres que utilizam anticoncepcional, com maior risco de pílulas com mais de 50 microgramas de estrogênio. Este estudo também demonstrou que mulheres tabagistas, usuárias destes métodos e com histórico de enxaqueca, têm aumento de três vezes no risco para a ocorrência de um AVC (Roach et al., 2015). O que torna essencial, na consulta de enfermagem, a investigação dos antecedentes pessoais dessas mulheres.

Entre as formas de avaliação do quadro clínico das mulheres nos estudos destacaram-se o uso de escalas de avaliação neurológica, com destaque para a National Institute of Health Stroke Scale, a Escala de Rankin Modificada, o Índice de Barthel Modificado e a Scandinavian Stroke Scale (Andersen \& Olsen, 2014; Antonio, Fuentes, Aguilar-Amat, \& Díez-Tejedor, 2011; Lima et al., 2017b). Todas essas escalas são validadas para uso no Brasil

Duas dessas escalas permitem investigar desde a severidade e a magnitude do déficit neurológico após o AVC, como a National Institute of Health Stroke Scale ou identificar comprometimento neurológico ou para predizer a mortalidade e morbidade, como a Scandinavian Stroke Scale (Brito et al., 2013; Luvizutto et al., 2012; Moro \& Pontes-Neto, 2009; Scandinavian Stroke Study Group, 1985).

Diante disso, é fundamental que o enfermeiro seja treinado para aplicar e interpretar os resultados dessas escalas no contexto agudo, pois seus resultados contribuem para o direcionamento da assistência que deverá ser implementada.

No contexto após o AVC, para determinar o potencial impacto sobre o cotidiano das pessoas acometidas, a Escala de Rankin Modificada é muito utilizada para determinar a extensão da incapacidade física após o AVC e a necessidade de assistência para realização de atividades instrumentais básicas da vida diária. Já o Índice de Barthel Modificado avalia o grau de independência para a realização de dez atividades de vida diária (Brito et al., 2013; Moro \& Pontes-Neto, 2009).

De modo geral, a aplicação dessas escalas em pacientes acometidas pelo AVC é de extrema relevância, pois possibilita avaliar a progressão dos déficits durante a internação, classificar a gravidade do evento e, partindo da avaliação inicial, realizar o planejamento da assistência pelo enfermeiro. Além de serem ferramentas para o acompanhamento da evolução da internação até a reabilitação pós alta, as escalas ainda geram dados que permitem a comparação entre as diferentes idades e a presença dos fatores de risco.

Isso possibilita o estudo de medidas de prevenção e reabilitação focalizadas para determinados grupos de risco, diminuindo a morbidade da população acometida pelo evento (Cechetti, Stuani, \& Paniz, 2013; Marques, Santos, Amaral, \& Paula, 2019). Logo, estudos como a publicação espanhola (Antonio, Fuentes, Aguilar-Amat, \& Díez-Tejedor, 2011) em que foram apresentados apenas os dados de alta, devem ser evitados, pois dificultam a comparação entre os resultados de admissão e de alta da paciente.

Nesse contexto, é importante destacar que entre os estudos selecionados as mulheres apresentaram moderada dependência após o AVC, independentemente de ter usado ou não os anticoncepcionais (Antonio, Fuentes, Aguilar-Amat, \& Díez-Tejedor, 2011; Lima et al., 2017b; Selvan et al., 2017). Esse processo pode dificultar seu processo de adaptação à vida, pois após o AVC, muitas das mulheres acompanhadas nos estudos incluídos na presente revisão, passaram a apresentar déficit sensitivo, motor, visual e/ou da fala, além de manifestações como migrânea, cefaleia, convulsão, vertigem e/ou tontura (Lima et al., 2017b; Selvan et al., 2017). De maneira geral, essas alterações ocorrem de maneira súbita e interferem no desempenho dos múltiplos papéis que as mulheres exercem, e em alguns casos, pode até mesmo ser necessário a implementação de cuidados básicos a intermediários da enfermagem (Ramos et al., 2020).

Por fim, publicações que abordassem a relação das manifestações clínicas de admissão e alta com o prognóstico das pacientes que sofreram o AVC não foram encontradas na literatura consultada. Isso ressalta que o registro isolado dos sinais e sintomas, como ocorreu em dois dos artigos (Maino, Siegerink, Algra, Peyvandi \& Rosendaal, 2016; Selvan et al., 2017), sem o uso de escalas, auxilia na caracterização dos quadros, mas torna-se inespecífico para comparações e avaliação da 
gravidade do evento e é, portanto, ineficiente para pesquisas, ou mesmo na assistência. Logo, incentivar o uso de indicadores clínicos na prática de enfermagem é essencial, pois eles permitem tanto a monitorar e acompanhar o quadro clínico dos pacientes, como avaliar a eficácia das intervenções que foram aplicadas.

\section{Limitações do estudo}

Primeiro, o método da revisão de escopo que fornece amplitude no lugar de análise aprofundada da qualidade metodológica das evidências identificadas. Segundo, houve restrição de idiomas para a inclusão dos artigos. A heterogeneidade dos estudos incluídos, com utilização de diferentes escalas, limitou a comparação entre os dados. Por fim, mesmo que duplas tenham trabalhado em cada etapa, não descartamos a subjetividade desse processo (viés de interpretação dos resultados).

\section{Contribuições para a prática}

O estudo traz contribuições para os enfermeiros da atenção básica, pois reforça a importância da investigação dos fatores de risco nas mulheres, pois há relação entre o uso de anticoncepcionais e a ocorrência ou recorrência do AVC. Para os enfermeiros que irão acompanhar essas pacientes após o AVC, seja na atenção básica ou nos ambulatórios de reabilitação neurológica, o estudo apresenta as manifestações clínicas mais comuns entre as usuárias de ACO, o que poderá contribuir para o planejamento da assistência de enfermagem. E por fim, o estudo apresenta diferentes escalas que permitem avaliar o quadro clínico após um episódio de AVC, seja na fase aguda ou crônica, o que poderá estimular o seu uso nas consultas e atendimentos, bem como maior reflexão sobre o tema.

\section{Considerações Finais}

Dentre os estudos analisados, o AVC em mulheres usuárias de anticoncepcional foi tão grave quanto o evento nas não usuárias. Apesar de nem todos os estudos detalharem a composição dos anticoncepcionais utilizados ou o tempo de uso anteriormente ao AVC, foi possível identificar que as mulheres que faziam uso dessa medicação apresentavam elevadas taxas de mortalidade, maior risco de desenvolver um novo AVC ou outro evento tromboembólico e, entre aquelas que usaram por menor tempo, menor gravidade do AVC.

Contudo, há escassez de estudos direcionados à avaliação da evolução clínica dessas mulheres, o que torna necessário o desenvolvimento de pesquisa nesta área, sendo esta uma sugestão das autoras para trabalhos futuros.

\section{Referências}

Andersen, K. K., \& Olsen, T. S. (2014). Severity of Stroke in Women Using Oral Contraceptives. Pharmacoepidemiology and Drug Safety, 23(S1), I-497. https://doi.org/10.1002/pds.3701

Antonio, L. A. R., Fuentes, B., Aguilar-Amat, M. J. \& Díez-Tejedor, E. (2011). "Píldora del día después" e ictus: un nuevo caso Emergency contraception and stroke. A new case report. Medicina Clínica, 136(14), 646-651. https://doi.org/10.1016/j.medcli.2010.05.020

Barker-Collo, S., Bennett, D. A., Krishnamurthi, R. V., Parmar, P., Feigin, V. L., Naghavi, M., \& Roth, G. A. (2015) Sex Differences in Stroke Incidence, Prevalence, Mortality and Disability-Adjusted Life Years: Results from the Global Burden of Disease Study 2013. Neuroepidemiology, 45(3), 203-214. https://doi.org/10.1159/000441103

Campos, C. J. G. (2004). Content analysis: a qualitative data analysis tool in health care. Revista Brasileira de Enfermagem, 57(5), 611-614. http://dx.doi.org/10.1590/S0034-71672004000500019

Champaloux, S. W., Tepper, N. K., Monsour, M., Curtis, K. M., Whiteman, M. K., Marchbanks, P. A., \& Jamieson, D. J. (2017). Use of combined hormonal contraceptives among women with migraines and risk of ischemic stroke. American Journal of Obstetrics and Gynecology, 216(5), 489.e1-489.e7. 10.1016/j.ajog.2016.12.019

Chang, B. P., Wira, C., Miller, J., Akhter, M., Barth, B. E., Willey, J. \& Madsen, T. (2017). Neurology Concepts: Young Women and Ischemic StrokeEvaluation and Management in the Emergency Department. Academic Emergency Medicine., 25(1), 54-64. https://doi.org/10.1111/acem.13243 
Hugon-Rodin, J., Gompel, A. \& Plu-Bureau, G. (2017). Epidemiology of hormonal contraceptives-related venous thromboembolism. European Journal of Endocrinology, 171(6), 221-230. 10.1530/EJE-14-0527

Li, F., Zhu, L., Zhang, J., He, H., Qin, Y., Cheng, Y., \& Xie, Z. (2019) Oral Contraceptive Use and Increased Risk of Stroke: A Dose-Response Meta-Analysis of Observational Studies. Frontiers in Neurology, 23(10), 993. https://doi.org/10.3389/fneur.2019.00993

Lillemoen, L., \& Pedersen, R. (2013). Ethical challenges and how to develop ethics support in primary health care. Nursing Ethics, 20(1), 96-108. https://doi.org/10.1177\%2F0969733012452687

Lima, A. C. S. (2017b). Efeitos do uso de anticoncepcionais hormonais combinados sobre o acidente vascular cerebral. (Tese de doutorado não publicada). Universidade Federal do Ceará, Fortaleza, CE.

Lima, A. C. S., Martins, L. C. G., Lopes, M. V. O., Araújo, T. L., Lima, F. E. T., Aquino, P. S. \& Moura, E. R. F. (2017a). Influence of hormonal contraceptives and the occurrence of stroke: integrative review. Revista Brasileira de Enfermagem, 70(3), 647-55. https://doi.org/10.1590/0034-7167-20160056

Madsen, T. E., Howard, G., Kleindorfer, D. O., Furie, K. L., Oparil, S., Manson, J. E., \& Howard, V. J. (2019). Sex Differences in Hypertension and Stroke Risk in the REGARDS Study: A Longitudinal Cohort Study. Hypertension, 74(4), 749-755. https://doi.org/10.1161/HYPERTENSIONAHA.119.12729

Maino, A., Siegerink, B., Algra, A., Peyvandi, F., \& Rosendaal, F. R. (2016) Recurrence and Mortality in Young Women With Myocardial Infarction or Ischemic Stroke: Long-term Follow-up of the Risk of Arterial Thrombosis in Relation to Oral Contraceptives (RATIO) Study. JAMA Internal Medicine, 176(1), 134-136. http://jamanetwork.com/article.aspx?doi=10.1001/jamainternmed.2015.6523

Melnyk, B. M., \& Fineout-Overholt, E. (2005). Making the case for evidence-based practice. In: Melnyk BM, Fineout-Overholt E. Evidence based practice in nursing \&amp; healthcare: a guide to best practice. Philadelphia: Lippincot Williams \&amp; Wilkins. 3-24.

Ministério da Saúde (BR), Secretaria de Vigilância em Saúde, Departamento de Vigilância de Doenças e Agravos Não Transmissíveis e Promoção da Saúde. (2018). Saúde Brasil Estados 2018: uma análise de situação de saúde segundo o perfil de mortalidade dos estados brasileiros e do Distrito Federal. Brasília: Ministério da Saúde, 428p. Recuperado de http://bvsms.saude.gov.br/bvs/publicacoes/saude_brasil_estados_2018_analise_situacao_saude_mortalidade.pdf

Pang, H., Fu, Q., Cao, Q., Hao, L. \& Zong, Z. (2019) Sex differences in risk factors for stroke in patients with hypertension and hyperhomocysteinemia. Scientific Reports, 9 (14313). 10.1038/s41598-019-50856-Z

Peters, M. D., Godfrey, C. M., Khalil, H., McInerney, P., Parker, D., \& Soares, C. B. (2015). Guidance for conducting systematic scoping reviews. International Journal of Evidence-Based Healthcare, 13(3), 141-146. https://doi.org/10.1097/xeb.0000000000000050

Poorthuis, M. H. F., Algra A. M., Algra A., Kappelle L. J. \& Klijn C. J. M. (2017). Female - and Male - Specific Risk Factors for Stroke. A Systematic Review and Meta-analysis. JAMA Neurology, 74(1), 75-81. 10.1001/jamaneurol.2016.3482

Roach, R. E., Helmerhorst, F. M., Lijfering, W. M., Stijnen, T., Algra, A., \& Dekkers, O. M. (2015). Combined oral contraceptives: the risk of myocardial infarction and ischemic stroke. Cochrane Database of Systematic Reviews, 15(8). https://doi.org/10.1002/14651858.CD011054.pub2

Salisbury, M., Pfeffer, G. \& Yip, S. (2011) Stroke in Young Women. Canadian Journal of Neurological Sciences, 38(3), 404-410. https://doi.org/10.1017/s0317167100011781

Selvan, P., Piran, P., Balucani, C., Tark, B., Adler, Z. \& Levine, S. R. (2017) Stroke and Etonogestrel/Ethinyl Estradiol Ring (NuvaRing): Clinical, Radiological, and Prognostic Features. Journal of Stroke \& Cerebrovascular Disease, 26(3), 608-617. https://doi.org/10.1016/j.jstrokecerebrovasdis.2016.11.111

Xu, Z., Li, Y., Tang, S., Huang, X. \& Chen, T. (2015). Current use of oral contraceptives and the risk of first-ever ischemic stroke: A meta-analysis of observational studies. Thrombosis Research, 136(1), 52-60. 10.1016/j.thromres.2015.04.021

Xu, Z., Yue, Y., Bai, J., Shen, C., Yang, J., Huang X., \& Li, Y. (2018). Association between oral contraceptives and risk of hemorrhagic stroke: a metaanalysis of observational studies. Archives of Gynecology and Obstetrics, 297(5), 1181-1191. https://doi.org/10.1007/s00404-018-4723-7 